\title{
Adsorption of S-Indacene on Silver Cluster — DFT Approach
}

\author{
B. Felcia Merlin ${ }^{a}$, R. Amrutha ${ }^{a, *}$ And P. Chandran ${ }^{b}$ \\ ${ }^{a}$ Department of Physics, KCG College of Technology, Chennai-600097, India \\ ${ }^{b}$ Department of Physics, Madras Christian College, Chennai-600059, India
}

Received: 17.02.2020 \& Accepted: 25.05.2020

Doi: $10.12693 /$ APhysPolA.138.447

*e-mail: amrutha@kcgcollege.com

\begin{abstract}
The investigation of the interfacial charge transfer across $\mathrm{S}$ (symmetric)-Indacene and silver metal cluster is performed with density functional theory calculations. The characterization of UV-visible and Raman spectra proves the existence of the redshift on adsorption of silver cluster, thus revealing a significant electrostatic molecule/metal interaction. The optimized structural geometry indicates bending of bonds in the cyclopentane ring near to the Ag cluster on adsorption. Frontier molecular orbitals point to the charge transfer at the organic-metal interface. Natural bond orbitals analysis evidences binding interactions through atomic orbital overlap of $\sigma$ electrons. This confirms the interfacial charge transfer from $\sigma(\mathrm{C}-\mathrm{C})$ to $\pi^{*}(\mathrm{Ag}-\mathrm{Ag})$ and $\pi(\mathrm{C}-\mathrm{C})$ to $\pi^{*}(\mathrm{Ag}-\mathrm{Ag})$, revealing the adsorption characteristics. The calculated reduction in electron reorganization energy, reduction in band gap and marked enhancement in first order hyperpolarizability in the adsorption of molecule/metal cluster indicates that the molecular system is a potential candidate for organic light-emitting diodes (OLEDs) and nonlinear optical (NLO) application. The charge transfer interaction was validated with molecular electrostatic potential and Fukui calculation.
\end{abstract}

topics: S-Indacene, UV-Vis, Raman, SERS

\section{Introduction}

Conjugated organic materials have remarkable application in the field of optoelectronics as potential semiconductors due to their efficient charge transport properties. S-Indacene, a planar cyclic conjugated hydrocarbon associated with aromatic and anti-aromatic character, possesses $12 \pi$ quasidelocalized electron systems $[1,2]$. The scientific interest in a silver cluster system and noble metal nanoparticles, such as silver and gold, plays a significant role in the field of nanoscale electronics because of their distinct optical, electrical and magnetic properties.

The metal cluster system is an intermediate state between atoms and bulk materials. It is considered as a model for surface-adsorbed molecule aiding for more insights in areas such as adsorption, lubrication, adhesion of dyes and corrosion protection [3, 4]. Rajkumar et al. [5, 6] investigated the adsorption functionalization on polycyclic aromatic hydrocarbons of linearly fused anthracene, tertacene and nonlinearly fused pyrene, chrysene on silver for the design of organic light-emitting diodes and nonlinear optical devices. Among the class of linear and nonliner fused polycyclic aromatic hydrocarbons, the S-Indacene constitutes an important category, typically due to its unique nature. Many studies reported that a rectilinear fused design of S-Indacene is highly reactive and unstable in the solid state. In the doped state, S-Indacene exhibits a thermally stable structure. The identification of this potential nature facilitates various applications such as semiconducting devices [7, 8]. Adsorption due to metal-molecule interaction enhances the intramolecular charge transfer (ICT) between a donor and acceptor. This characteristic acts as the key parameter in determining the way the conductivity influences the performance and efficiency of organic light-emitting diodes [9]. Extensive analyses have shown that conjugated organic materials have greater nonlinear optical response, higher nonlinear susceptibility and facile architectural modification as compared to inorganic materials. This is caused by the D-A chromospheres. Conjugated materials with additional electron withdrawing or electron donating groups enhance a high charge carrier mobility and reduced HOMO-LUMO levels, which are the essential requirement for the rational design of organic light-emitting diodes and nonlinear optical devices. Hence, they are used in optical telecommunications, signal processing and image transmission [1-10].

Various studies on acene-like structures have reported that indenoflourenes have been used as an ambipolar charge transport material, indacenodi(benzothiophene)s as a hole/electron transport material in organic field-effect transistors (OFETs). The interaction of fused rings results in improving electrical conductivity and charge carrier mobility 
in the solid state [11]. Shi et al. [12] have synthesized bisindeno-[n]thienoacenes using spectroscopic techniques. They explored their application in the design of photoactive and electroactive materials.

Growth behaviour of S-Indacene attached to two carbonyl groups on $\mathrm{Ag}$ (110) surface results in twodimensional (2D) ordered structures, generating interest in the investigation of a growth mechanism of organic semiconductors on metal [13]. Zarate et al. [8] investigated theoretically the interaction of various transition metals $\mathrm{Fe}, \mathrm{Co}, \mathrm{Ni}, \mathrm{Ru}, \mathrm{Rh}$, Re and Mn connected as bi-metallic centres to S-Indacene. The analysis confirms these complexes involve a charge transfer from S-Indacene to metal and the atomic charges are delocalized in the metallic centres upon oxidation. Keeping all that in mind, in the present work quantum chemical calculations were carried out to investigate the adsorption characteristics of S-Indacene on a trimer silver cluster and their vibrational spectra and the associated charge transfer are analyzed.

\section{Computational study}

In the present investigation, theoretical calculations to investigate the reorganization energy were carried out with DFT using the B3LYP/6$311+\mathrm{G}(\mathrm{d}, \mathrm{p})$ method. Adsorption mechanism of S-Indacene on silver (Ag-S-Indacene) and Raman spectra analysis were performed at B3PW91 functional and LANL2DZ basis set, using a commercially available Gaussian 09 package. Previous studies reported B3PW91 functional and LANL2DZ basis set as an effective standard choice for larger molecular systems involving metals [14]. Although the B3LYP hybrid functional was successfully applied for predicting molecular properties, it fails to attain the electron gas limit. The uniform homogeneous electron gas limit was achieved by B3PW91 functional [15]. From the theoretical and experimental standpoint, the growth pattern of trimer silver cluster $\mathrm{Ag}_{3}$ is considered to be best suited for the adsorption mechanism. Various studies reported that $\mathrm{Ag}_{3}$ has a low energy gap and significant optical absorption spectrum in contribution to the behaviour of metallic surface. Moreover, it was identified that $\mathrm{Ag}_{3}$ is more stable and reactive, and has a great contribution to the material science research [16]. In order to obtain good results, computations on ground state geometry optimization were carried out for different tilted orientations and the most stable geometry was used for further investigations. Convergence of the molecular geometry calculations was confirmed and no imaginary frequency values were observed. The molecular geometry optimization, UV-Vis characterization spectrum was simulated using DFT/B3PW91/LANL2DZ and TD-DFT/B3PW91/LANL2DZ. The plot of frontier molecular orbitals, molecular electrostatic potential of S-Indacene and Ag-S-Indacene were visualized. Further, the charge transfer mechanism

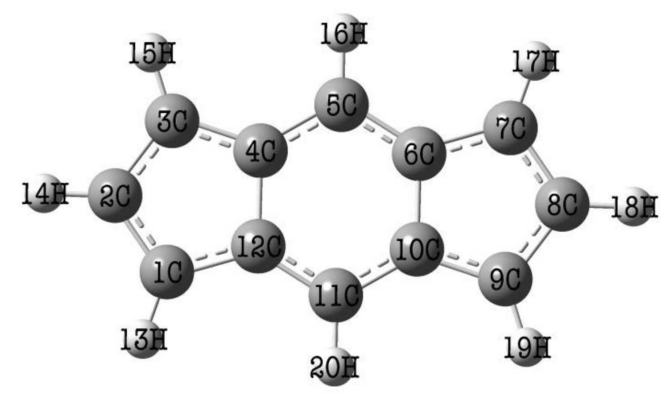

Fig. 1. Optimized geometry of S-Indacene at DFT/B3LYP/6-311+G (d,p) level of theory.

TABLE I

The calculated hole and electron reorganization energy using B3LYP/6-311+G (d,p).

\begin{tabular}{c|c|c|c}
\hline \hline Molecule & $\begin{array}{c}\text { S-Indacene } \\
{[\mathrm{eV}]}\end{array}$ & Molecule & $\begin{array}{c}\text { S-Indacene } \\
{[\mathrm{eV}]}\end{array}$ \\
\hline $\mathrm{IPv}$ & 7.21 & $\mathrm{EAa}$ & 1.82 \\
$\mathrm{IPa}$ & 7.06 & $\mathrm{EEP}$ & 1.85 \\
$\mathrm{HEP}$ & 6.94 & $\lambda_{h}$ & 0.27 \\
$\mathrm{EAv}$ & 1.74 & $\lambda_{e}$ & 0.11
\end{tabular}

was discussed by the natural population analysis (NPA) and natural bond orbital (NBO) analysis. In the case of Fukui calculations, they were based on the natural population analysis by carrying out a single point energy calculation of 0 , +1 and -1 , namely neutral, cationic and anionic species of the molecular systems.

\section{Results and discussion}

\subsection{Molecular geometry} and charge transfer analysis

To investigate the suitability of the molecular system for the rational design of OLEDs, reorganization energy calculations were carried out at the DFT level, using B3LYP /6-311+G(d,p) method which was shown in Table I. The optimized geometry of the molecule at DFT/B3LYP /6-311+G(d,p) method was shown in Fig. 1.

Generally, the transport ability of hole and electron can be represented as:

$$
\begin{gathered}
\lambda_{\text {hole }}=\lambda_{+}-\lambda_{-}=\left[E^{\text {ion }}\left(Q_{n}\right)-E^{0}\left(Q_{n}\right)\right] \\
+\left[E^{\text {ion }}\left(Q_{c}\right)-E^{0}\left(Q_{c}\right)\right]=\mathrm{IP}_{\mathrm{v}}-\mathrm{HEP}, \\
\lambda_{\text {electron }}=\lambda_{-}-\lambda_{0}=\left[E^{\mathrm{ion}}\left(Q_{n}\right)-E^{0}\left(Q_{n}\right)\right] \\
-\left[E^{\mathrm{ion}}\left(Q_{c}\right)-E^{0}\left(Q_{c}\right)\right]=\mathrm{EEP}-\mathrm{EA}_{\mathrm{v}},
\end{gathered}
$$

where $E^{\text {ion }}\left(Q_{n}\right)$ and $E^{\text {ion }}\left(Q_{c}\right)$ is the energy of the neutral and charged molecule in the cationic and anionic geometry, respectively. In the ground state, the respective energy is denoted as $E^{0}\left(Q_{n}\right)$ and $E^{0}\left(Q_{c}\right)$. 


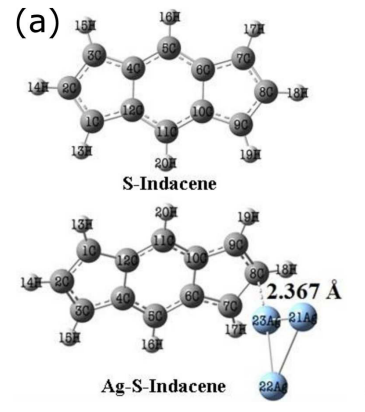

(b)

(c)

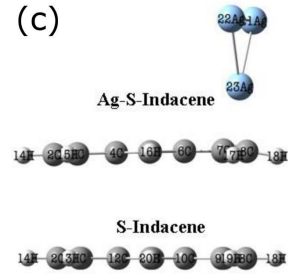

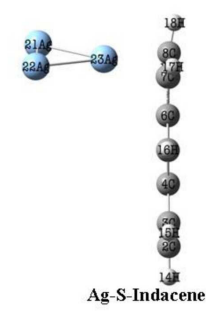
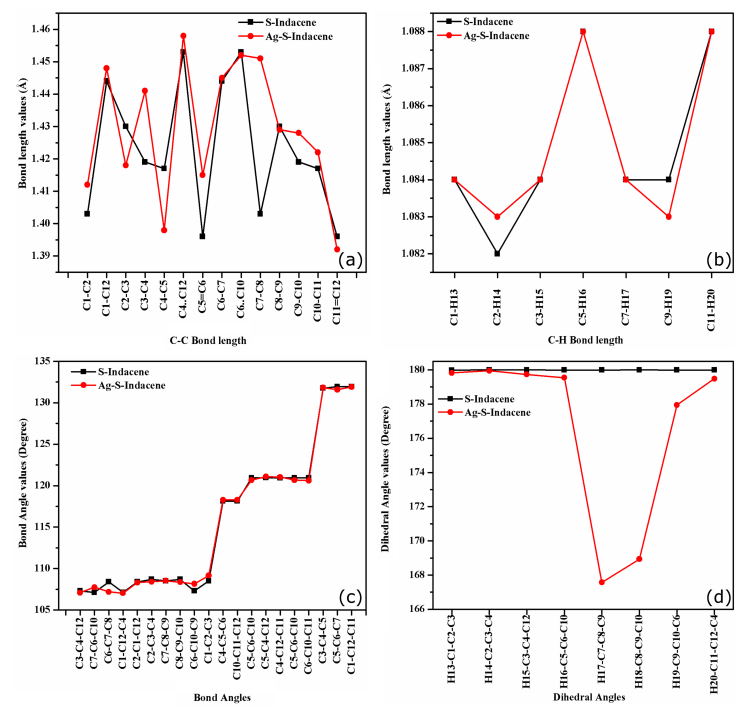

Fig. 3. (a) Structural parameters variation graph of $\mathrm{C}-\mathrm{C}$ bond length values of S-Indacene and Ag-S-Indacene systems. (b) The structural parameters variation graph of $\mathrm{C}-\mathrm{H}$ bond length values of S-Indacene and Ag-S-Indacene systems. (c) The structural parameters variation graph of $\mathrm{C}-\mathrm{C}-\mathrm{C}$ bond angle values of S-Indacene and $\mathrm{Ag}$-S-Indacene systems. (d) The structural parameters variation graph of selected dihedral angle values of S-Indacene and Ag-S-Indacene systems.

According to Marcus theory, the high chargetransfer takes place when $\lambda$ value becomes lesser. Our calculation has shown that the electron reorganization energy is smaller than hole reorganization energy and therefore this rectilinear fused threering system can be used as electron transport layers (ETL) in OLED devices. The calculated ground state energies of S-Indacene structure in the optimized $\mathrm{C}_{2 h}$ geometry on the Gaussian basis set at STO-3G, 3-21G, 4-31G, DZV are $-1.233 \times 10^{4} \mathrm{eV}$, $-1.241 \times 10^{4} \mathrm{eV},-1.247 \times 10^{4} \mathrm{eV},-1.2478 \times 10^{4} \mathrm{eV}$, respectively [17].

The optimized gas phase molecular geometry of S-Indacene before and after the adsorption of the silver cluster (S-Indacene and Ag-S-Indacene) was shown in Fig. $2 \mathrm{a}-\mathrm{c}$. Importantly, the shortest distance between the silver atom Ag23 and carbon atom C8 in Ag-S-Indacene system was found to be $2.367 \AA$, which was also noted in Fig. 2a. The global minimum ground state energy of S-Indacene molecule with B3PW91/LANL2DZ basis set was equal to $-1.256 \times 10^{4} \mathrm{eV}$. After adsorption the minimum energy of Ag-S-Indacene was reduced to $-2.446 \times 10^{4} \mathrm{eV}$. As it is shown in Fig. 3a, the significant variations were noticed in $\mathrm{C}-\mathrm{C}$ bond lengths values near to the metal cluster C7-C8 (1.403 $\AA$ to $1.451 \AA$ ) and C9-C10 (1.419 $\AA$ to $1.428 \AA$ ). The $\mathrm{C}-\mathrm{C}$ bond length values near to the metal cluster are found to be pointedly lesser when compared to the remaining $\mathrm{C}-\mathrm{C}$ bond lengths (C1-C12, C3-C4, C4... C12, C6-C7, C6-C10, C8-C9) that are calculated to be $1.448 \AA$, $1.441 \AA, 1.458 \AA, 1.445 \AA, 1.452 \AA, 1.452 \AA$,

respectively. This change gives rise to a prominent distortion in the geometry of the cyclopentane ring near to the silver cluster. The $\mathrm{C} 2-\mathrm{C} 3$, C4... C5, $\mathrm{C} 11=\mathrm{C} 12$ bond lengths are $1.418 \AA$, $1.398 \AA, 1.392 \AA$, under lesser alterations. Moreover, the $\mathrm{C}-\mathrm{H}$ bond length values of S-Indacene remain the same both before and after the adsorption of the Ag cluster. Figure $3 \mathrm{~b}$ confirms this effect. In [18], it was reported that the adsorption of the bare silver cluster $\mathrm{Ag}_{3}$ results in a significant reduction of $\mathrm{Ag}-\mathrm{Ag}$ bond length (3.164 $\AA$ to $2.905 \AA$ ). In our investigation, one can notice that a variation in $\mathrm{C}-\mathrm{C}, \mathrm{C}-\mathrm{H}$ bond lengths, and the electron affinity of the $\mathrm{Ag}$ cluster links the metal and molecule and confirms the charge transfer along the substrate. In Fig. $3 \mathrm{c}$ and $\mathrm{d}$, the bond angles of S-Indacene in proximity to the $\mathrm{Ag}$ atoms are $108^{\circ}$ (C7-C8-C9; C8-C9-C10) and their corresponding dihedral angles are at $167^{\circ}$ (H17-C7-C8C9) and $168^{\circ}$ (H18-C8-C9-C10). These deviations in the bond angle and dihedral angle indicate without doubt a break in the planar cyclic conjugated symmetry of S-Indacene that affects the bending of bonds in the cyclopentane ring near to the $\mathrm{Ag}$ cluster on adsorption.

\subsection{Electronic properties:}

\section{HOMO and LUMO and MEP analysis}

The highest molecular orbital energy (HOMO) defines the ability of electron giving and lowest unoccupied molecular orbital (LUMO) energy 


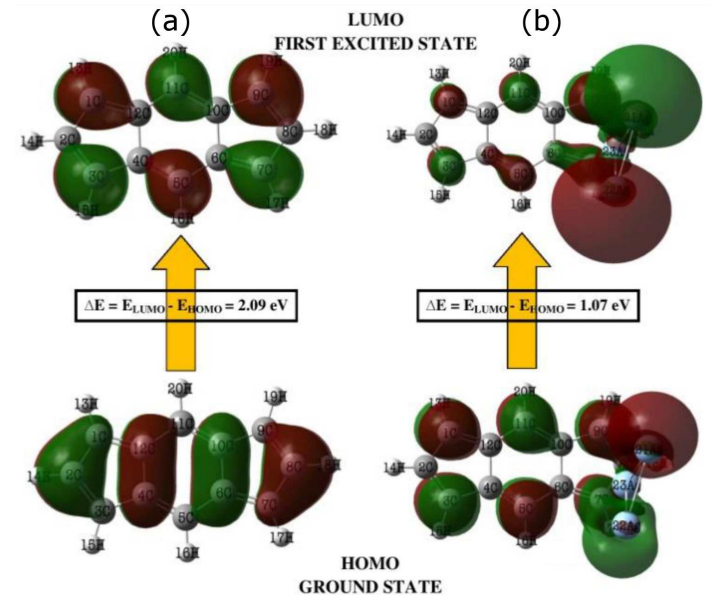

Fig. 4. FMOs obtained for (a) S-Indacene, and (b) Ag-S-Indacene systems.

characterizes the ability of electron accepting [19]. The frontier molecular orbitals play an important role in the electronic and optical properties [20]. The HOMO-LUMO energy gap helps to explain the stability and reactivity of the molecule, which was an important parameter in analyzing its electronic properties. A molecule with a small energy gap is usually shows a low kinetic stability, high chemical reactivity and is also said to be soft [21]. In general, the energy gap of the $\pi$-conjugated materials can be tuned by the chemical modifications for improving the performance of optoelectronic devices [22].

In frontier molecular orbital diagram, red colour is indicated as a positive phase and green colour is indicated as a negative phase. The HOMO and LUMO of S-Indacene and Ag-S-Indacene are presented in Fig. $4 \mathrm{a}$ and b. It is evident from the molecular orbitals that HOMO is located mainly around the carbon atoms of the benzene ring that are in proximity of cyclopentane. The other two carbon atoms at the top and bottom of the benzene ring are influenced to a lesser degree. The LUMO tends to form specific antibonding orbitals at the top and bottom situated carbon atoms of S-Indacene. In the case of Ag-S-Indacene, on adsorption the HOMOs are located effectively at the top and bottom situated carbon atoms of S-Indacene and extended at the surface of $\mathrm{Ag}$ atoms favouring a better surface metalmolecule interaction. The LUMOs are located effectively at the top and bottom carbon atoms of S-Indacene and extended well over the Ag cluster. This is due to the interfacial charge transfer going on in the organic-metal interface due to overlapping of $\sigma$ orbitals of top and bottom carbon atoms of S-Indacene and valence orbitals of $\mathrm{Ag}$ atoms. It is evident from the shifting of molecular orbitals from S-Indacene overlapped with cyclopentane ring and silver cluster leading to adsorption. The comparison of the charge transfer analysis of linearly fused two-ring naphthalene, three-ring
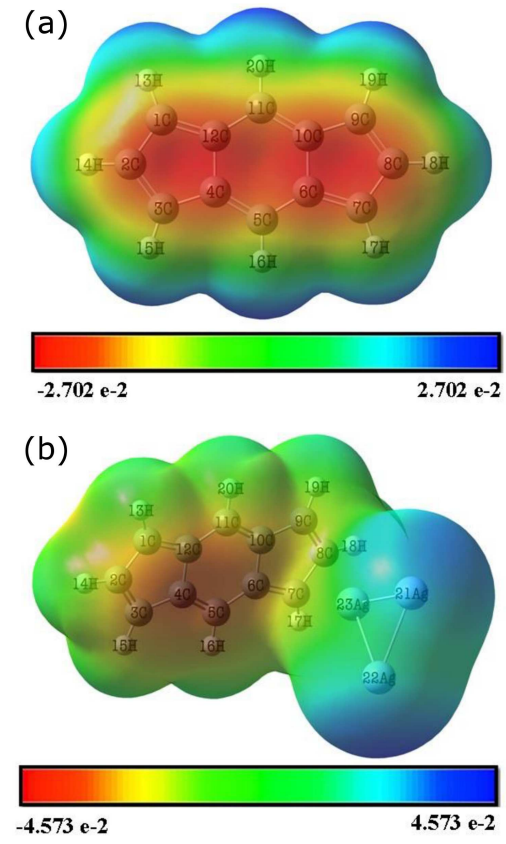

Fig. 5. MEP plots obtained for (a) S-Indacene, and (b) Ag-S-Indacene.

anthracene and four-ring tetracene with the $\mathrm{Ag}_{3}$ cluster shows a strong intramolecular charge transfer $[5,23,24]$. Hence from the findings, it is identified that $\mathrm{S}$-Indacene- $\mathrm{Ag}_{3}$ is unique in nature, showing interfacial charge transfer when reacted with silver. The band gap of S-Indacene is $2.09 \mathrm{eV}$ and is noticeably reduced after the adsorption of silver of $1.07 \mathrm{eV}$ energy. The calculated smaller energy gap value of the investigated molecular system is more favourable for the electronic transition. The decrease in energy gap value in the molecular system indicates the flow of charge carriers between the investigated systems and results in the increase in conductivity. The reduction in band gap along with the enhancement in the HOMO value $(4.19 \mathrm{eV})$ of $\mathrm{Ag}$-S-Indacene results in the increase in first order hyperpolarizability. The LUMO value of Ag-S-Indacene is $3.128 \mathrm{eV}$.

Molecular electrostatic potential (MEP) plot clearly explains the site for chemical reactivity of the molecular system. It gives information about the total electrostatic effect at any given point around the molecule influenced by the total charge density distribution [25]. The values of electrostatic potential are denoted by different colour gradients in the order: red less than orange, less than yellow, less than green, less than blue. Red colour represents the region of the most negative electrostatic potential region (electrophilic site). Blue colour represents the region of the most positive electrostatic potential region (nucleophilic site) and green colour represents the zero potential region [26]. To illustrate the reactivity sites and polarity of S-Indacene and Ag-S-Indacene, their corresponding molecular electrostatic potential surface with an electron density isovalue of $0.0004 \mathrm{a} . \mathrm{u}$ 
are shown in Fig. 5a and b. The molecular electrostatic potential surface of S-Indacene indicates that the electronegative potential (red colour) present over the carbon atoms of S-Indacene corresponds to the favourable electrophilic site. The electropositive potential (green colour) surrounding the hydrogen atom corresponds to the favourable nucleophilic site. After adsorption, redistribution of electron density is seen from the reduction in the electrostatic negative potential charges in the carbon atoms of cyclopentane ring near to the silver cluster overlapped with the electrostatic positive potential around the $\mathrm{Ag}$ atoms. It is because of the strong surface interaction between S-Indacene and silver associated with reliable transfer of charges from S-Indacene to silver cluster. It is also evident with the shifting of molecular orbitals from S-Indacene overlapped with cyclopentane ring and silver cluster.

\subsection{Natural bond orbital analysis}

Natural bond orbital (NBO) analysis is an efficient tool for investigating intra- and intermolecular interactions of the molecular systems, and it extensively explains the charge transfer interaction between the filled orbital (bond or lone pair) and unfilled orbital (anti-bonding) by means of second order perturbation theory [27]. A value of larger second order perturbation interaction energy $E^{(2)}$ indicates there is an extreme interaction between electron donors and electron acceptors, i.e., leading to higher donating ability from electron donors to electron acceptors resulting to a greater extent of conjugation in the whole system [28]. The stabilization energy can be calculated with

$$
E^{(2)}=\Delta E_{i j}=q_{i} \frac{F(i, j)^{2}}{\varepsilon_{i}-\varepsilon_{j}},
$$

where $q_{i}$ is the $i^{\text {th }}$ donor orbital occupancy, $\varepsilon_{i}$ and $\varepsilon_{j}$ are the diagonal elements and $F(i, j)$ is the off diagonal NBO fock matrix element. NBO clearly explains the inter- and intramolecular interactions by the mathematically chosen possibility of all the orbitals occupying the highest percentage of electron density. The stabilized donor-acceptor interaction resulting in electron density delocalization between occupied and unoccupied natural Lewis structure is properly explained. Theoretically computed NBO values performed on S-Indacene and Ag-S-Indacene were listed in Table II. Higher $E^{(2)}$ of S-Indacene basically originates from the atomic overlap between $\sigma(\mathrm{C}-\mathrm{C})$ to $\sigma^{*}(\mathrm{C}-\mathrm{C})$ orbitals confirming higher intermolecular charge transfer within the molecule. This is identified with increased electron density stabilizing the system and correlated well with molecular electrostatic potential plot of S-Indacene. In the case of Ag-S-Indacene, on adsorption the molecular interactions are identified from the orbital overlap between $\sigma(\mathrm{C}-\mathrm{C})$ to $\pi^{*}(\mathrm{Ag}-\mathrm{Ag})$. The overlap of orbitals is between Ag21, Ag22 and $\sigma$ orbitals of S-Indacene. This ac-

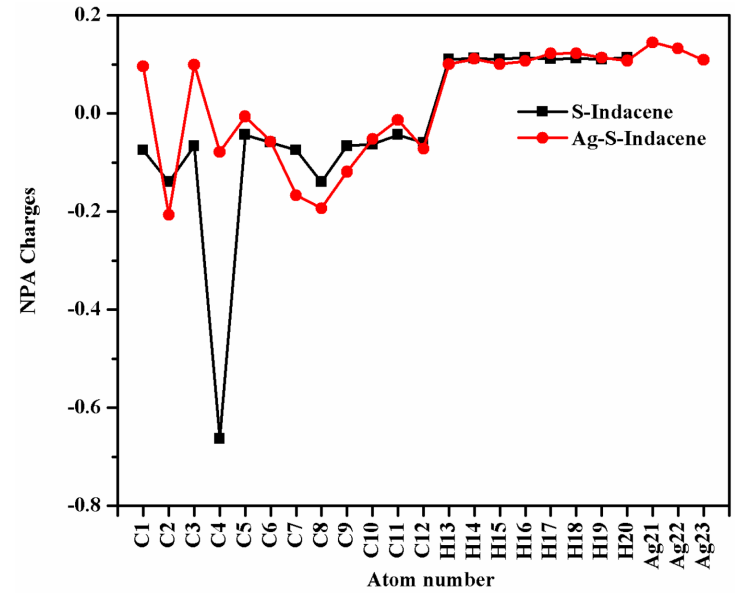

Fig. 6. The natural population atomic charge values variation graph of S-Indacene and Ag-S-Indacene systems.

counts for hyperconjugative or $\sigma$ conjugation interactions involving charge transfer from $\sigma$ bonding donor to $\pi^{*}$ antibonding acceptor extended molecular orbitals establishing a strong surface binding interaction. These hyper conjugative interactions recognized through NBO analysis very soundly correlate with the shifting of molecular orbitals from S-Indacene overlapped with cyclopentane ring and silver cluster. Comparing the NBO analysis of linearly fused two-ring naphthalene, threering anthracene, four-ring tetracene with the $\mathrm{Ag}_{3}$ cluster, the orbital overlap takes place between $\pi(\mathrm{C}-\mathrm{C})$ to $\pi^{*}(\mathrm{C}-\mathrm{C})$ and $\pi(\mathrm{C}-\mathrm{C})$ to $\sigma^{*}(\mathrm{Ag}-\mathrm{Ag})$ orbitals $[5,23,24]$. This confirms that S-Indacene shows a unique nature when reacted with silver.

The method of natural population analysis is an effective measure to determine the electronic configuration and to calculate the net charge associated with each atom in a polyatomic molecule. It also describes the difference in charges which are distributed within the subshells of atomic orbitals of each atom associated with its total electron population [28]. In order to analyze the charge transfer between S-Indacene and silver, the atomic charges of S-Indacene and Ag-S-Indacene are presented in Fig. 6.

The carbon atoms of S-Indacene are negatively charged and hydrogen atoms are positively charged. However, after the adsorption on silver, the carbon atoms which are away from silver atoms (C4, C5, C6, C10, C11, C12) are more negatively charged. Correspondingly, the carbon atoms closer to silver atoms (C7, C8, C9) are less negatively charged and the Ag atoms (Ag21, Ag22, Ag23) are significantly less positive in nature. These theoretically calculated values indicate an enhanced surface level interaction between the molecule and metal confirming a reliable charge transfer from S-Indacene to silver. These findings correlate well with the overlapping of molecular orbitals between S-Indacene and the silver cluster. 
TABLE II

Second order perturbation theory analysis of Fock matrix of S-Indacene and Ag-S-Indacene systems by NBO analysis

\begin{tabular}{|c|c|c|c|c|c|}
\hline & Donor $(i)$ & Acceptor $(j)$ & $\begin{array}{c}E(2) \\
{[\mathrm{Kcal} / \mathrm{mol}]}\end{array}$ & $E(j)-E(i)$ & $F(i, j)$ \\
\hline \multirow[t]{39}{*}{ S-Indacene } & \multirow[t]{2}{*}{$\pi(\mathrm{C} 1-\mathrm{C} 2)$} & $\pi^{*}(\mathrm{C} 4-\mathrm{C} 5)$ & 1.05 & 1.21 & 0.032 \\
\hline & & $\pi^{*}(\mathrm{C} 11-\mathrm{C} 12)$ & 6.65 & 1.24 & 0.081 \\
\hline & \multirow[t]{2}{*}{$\sigma(\mathrm{C} 1-\mathrm{C} 2)$} & $\sigma^{*}(\mathrm{C} 3-\mathrm{C} 4)$ & 25.01 & 0.28 & 0.077 \\
\hline & & $\sigma^{*}(\mathrm{C} 11-\mathrm{C} 12)$ & 16.56 & 0.29 & 0.062 \\
\hline & \multirow[t]{2}{*}{$\pi(\mathrm{C} 1-\mathrm{C} 12)$} & $\pi^{*}(\mathrm{C} 4-\mathrm{C} 5)$ & 5.12 & 1.19 & 0.070 \\
\hline & & $\pi^{*}(\mathrm{C} 10-\mathrm{C} 11)$ & 2.89 & 1.19 & 0.052 \\
\hline & \multirow[t]{2}{*}{$\pi(\mathrm{C} 2-\mathrm{C} 3)$} & $\pi^{*}(\mathrm{C} 3-\mathrm{C} 4)$ & 1.17 & 1.19 & 0.033 \\
\hline & & $\pi^{*}(\mathrm{C} 4-\mathrm{C} 5)$ & 7.18 & 1.19 & 0.083 \\
\hline & \multirow[t]{3}{*}{$\sigma(\mathrm{C} 3-\mathrm{C} 4)$} & $\sigma^{*}(\mathrm{C} 1-\mathrm{C} 2)$ & 16.98 & 0.27 & 0.062 \\
\hline & & $\sigma^{*}(\mathrm{C} 5-\mathrm{C} 6)$ & 28.58 & 0.28 & 0.082 \\
\hline & & $\sigma^{*}(\mathrm{C} 11-\mathrm{C} 12)$ & 20.19 & 0.28 & 0.069 \\
\hline & \multirow[t]{2}{*}{$\pi(\mathrm{C} 4-\mathrm{C} 5)$} & $\pi^{*}(\mathrm{C} 3-\mathrm{C} 3)$ & 2.51 & 1.22 & 0.049 \\
\hline & & $\pi^{*}(\mathrm{C} 6-\mathrm{C} 7)$ & 5.94 & 1.19 & 0.075 \\
\hline & \multirow[t]{2}{*}{$\pi(\mathrm{C} 4-\mathrm{C} 12)$} & $\pi^{*}(\mathrm{C} 4-\mathrm{C} 5)$ & 2.17 & 1.16 & 0.045 \\
\hline & & $\pi^{*}(\mathrm{C} 11-\mathrm{C} 12)$ & 2.45 & 1.19 & 0.049 \\
\hline & \multirow[t]{2}{*}{$\pi(\mathrm{C} 5-\mathrm{C} 6)$} & $\pi^{*}(\mathrm{C} 3-\mathrm{C} 4)$ & 5.40 & 1.23 & 0.073 \\
\hline & & $\pi^{*}(\mathrm{C} 6-\mathrm{C} 7)$ & 2.40 & 1.20 & 0.048 \\
\hline & \multirow[t]{3}{*}{$\sigma(\mathrm{C} 5-\mathrm{C} 6)$} & $\sigma^{*}(\mathrm{C} 3-\mathrm{C} 4)$ & 16.72 & 0.28 & 0.062 \\
\hline & & $\sigma^{*}(\mathrm{C} 7-\mathrm{C} 8)$ & 22.58 & 0.28 & 0.072 \\
\hline & & $\sigma^{*}(\mathrm{C} 9-\mathrm{C} 10)$ & 19.14 & 0.29 & 0.066 \\
\hline & \multirow[t]{2}{*}{$\pi(\mathrm{C} 6-\mathrm{C} 7)$} & $\pi^{*}(\mathrm{C} 4-\mathrm{C} 5)$ & 2.89 & 1.19 & 0.052 \\
\hline & & $\pi^{*}(\mathrm{C} 10-\mathrm{C} 11)$ & 5.12 & 1.19 & 0.070 \\
\hline & $\pi(\mathrm{C} 6-\mathrm{C} 10)$ & $\pi^{*}(\mathrm{C} 5-\mathrm{C} 6)$ & 2.45 & 1.19 & 0.049 \\
\hline & $\pi(\mathrm{C} 7-\mathrm{C} 8)$ & $\pi^{*}(\mathrm{C} 5-\mathrm{C} 6)$ & 6.65 & 1.24 & 0.081 \\
\hline & \multirow[t]{2}{*}{$\sigma(\mathrm{C} 7-\mathrm{C} 8)$} & $\sigma^{*}(\mathrm{C} 5-\mathrm{C} 6)$ & 16.56 & 0.29 & 0.062 \\
\hline & & $\sigma^{*}(\mathrm{C} 9-\mathrm{C} 10)$ & 25.01 & 0.28 & 0.077 \\
\hline & \multirow[t]{2}{*}{$\pi(\mathrm{C} 8-\mathrm{C} 9)$} & $\pi^{*}(\mathrm{C} 9-\mathrm{C} 10)$ & 1.17 & 1.19 & 0.033 \\
\hline & & $\pi^{*}(\mathrm{C} 10-\mathrm{C} 10)$ & 7.18 & 1.19 & 0.083 \\
\hline & \multirow[t]{2}{*}{$\pi(\mathrm{C} 9-\mathrm{C} 10)$} & $\pi^{*}(\mathrm{C} 5-\mathrm{C} 6)$ & 5.03 & 1.23 & 0.070 \\
\hline & & $\pi^{*}(\mathrm{C} 10-\mathrm{C} 11)$ & 2.64 & 1.20 & 0.050 \\
\hline & \multirow[t]{3}{*}{$\sigma(\mathrm{C} 9-\mathrm{C} 10)$} & $\sigma^{*}(\mathrm{C} 5-\mathrm{C} 6)$ & 20.19 & 0.28 & 0.069 \\
\hline & & $\sigma^{*}(\mathrm{C} 7-\mathrm{C} 8)$ & 16.98 & 0.27 & 0.062 \\
\hline & & $\sigma^{*}(\mathrm{C} 11-\mathrm{C} 12)$ & 28.58 & 0.28 & 0.082 \\
\hline & \multirow[t]{2}{*}{$\pi(\mathrm{C} 10-\mathrm{C} 11)$} & $\pi^{*}(\mathrm{C} 1-\mathrm{C} 12)$ & 5.94 & 1.19 & 0.075 \\
\hline & & $\pi^{*}(\mathrm{C} 9-\mathrm{C} 10)$ & 2.51 & 1.22 & 0.049 \\
\hline & \multirow[t]{2}{*}{$\pi(\mathrm{C} 11-\mathrm{C} 12)$} & $\pi^{*}(\mathrm{C} 1-\mathrm{C} 12)$ & 2.40 & 1.20 & 0.048 \\
\hline & & $\pi^{*}(\mathrm{C} 9-\mathrm{C} 10)$ & 5.40 & 1.23 & 0.073 \\
\hline & \multirow[t]{2}{*}{$\sigma(\mathrm{C} 9-\mathrm{C} 10)$} & $\sigma^{*}(\mathrm{C} 3-\mathrm{C} 4)$ & 19.14 & 0.28 & 0.066 \\
\hline & & $\sigma^{*}(\mathrm{C} 9-\mathrm{C} 10)$ & 16.72 & 0.28 & 0.062 \\
\hline \multirow[t]{5}{*}{ Ag-S-Indacene } & $\pi(\mathrm{C} 1-\mathrm{C} 2)$ & $\pi^{*}(\mathrm{C} 11-\mathrm{C} 12)$ & 3.25 & 1.24 & 0.080 \\
\hline & $\sigma(\mathrm{C} 1-\mathrm{C} 2)$ & $\sigma^{*}(\mathrm{C} 11-\mathrm{C} 12)$ & 5.31 & 0.28 & 0.051 \\
\hline & $\pi(\mathrm{C} 1-\mathrm{C} 12)$ & $\pi^{*}(\mathrm{C} 4-\mathrm{C} 5)$ & 2.53 & 1.21 & 0.070 \\
\hline & $\pi(\mathrm{C} 2-\mathrm{C} 3)$ & $\pi^{*}(\mathrm{C} 4-\mathrm{C} 5)$ & 3.32 & 1.23 & 0.081 \\
\hline & $\pi(\mathrm{C} 3-\mathrm{C} 4)$ & $\pi^{*}(\mathrm{C} 11-\mathrm{C} 12)$ & 2.52 & 1.22 & 0.070 \\
\hline
\end{tabular}


TABLE II (cont.)

\begin{tabular}{|c|c|c|c|c|c|}
\hline & Donor $(i)$ & Acceptor $(j)$ & $\begin{array}{c}E(2) \\
{[\mathrm{Kcal} / \mathrm{mol}]}\end{array}$ & $E(j)-E(i)$ & $F(i, j)$ \\
\hline \multirow[t]{18}{*}{ Ag-S-Indacene } & $\pi(\mathrm{C} 4-\mathrm{C} 5)$ & $\pi^{*}(\mathrm{C} 6-\mathrm{C} 7)$ & 2.95 & 1.18 & 0.074 \\
\hline & $\sigma(\mathrm{C} 4-\mathrm{C} 5)$ & $\sigma^{*}(\mathrm{C} 6-\mathrm{C} 7)$ & 9.76 & 0.25 & 0.065 \\
\hline & $\pi(\mathrm{C} 4-\mathrm{C} 12)$ & $\pi^{*}(\mathrm{C} 11-\mathrm{C} 12)$ & 1.20 & 1.20 & 0.048 \\
\hline & $\pi(\mathrm{C} 5-\mathrm{C} 6)$ & $\pi^{*}(\mathrm{C} 3-\mathrm{C} 4)$ & 2.83 & 1.19 & 0.073 \\
\hline & $\pi(\mathrm{C} 6-\mathrm{C} 7)$ & $\pi^{*}(\mathrm{C} 10-\mathrm{C} 11)$ & 2.51 & 1.20 & 0.069 \\
\hline & $\sigma(\mathrm{C} 6-\mathrm{C} 7)$ & $\sigma^{*}(\mathrm{C} 8-\mathrm{C} 9)$ & 11.46 & 0.25 & 0.070 \\
\hline & & $\pi^{*}(\mathrm{Ag} 21-\mathrm{Ag} 22)$ & 0.04 & 0.33 & 0.005 \\
\hline & $\pi(\mathrm{C} 6-\mathrm{C} 10)$ & $\pi^{*}(\mathrm{C} 5-\mathrm{C} 6)$ & 1.14 & 1.18 & 0.046 \\
\hline & $\pi(\mathrm{C} 7-\mathrm{C} 8)$ & $\pi^{*}(\mathrm{C} 5-\mathrm{C} 6)$ & 3.44 & 1.21 & 0.082 \\
\hline & $\pi(\mathrm{C} 8-\mathrm{C} 9)$ & $\pi^{*}(\mathrm{C} 10-\mathrm{C} 11)$ & 3.45 & 1.21 & 0.082 \\
\hline & & $\pi^{*}(\mathrm{Ag} 21-\mathrm{Ag} 22)$ & 0.03 & 0.74 & 0.006 \\
\hline & $\sigma(\mathrm{C} 8-\mathrm{C} 9)$ & $\sigma^{*}(\mathrm{C} 6-\mathrm{C} 7)$ & 10.10 & 0.28 & 0.071 \\
\hline & & $\pi^{*}(\mathrm{Ag} 21-\mathrm{Ag} 22)$ & 0.03 & 0.34 & 0.005 \\
\hline & $\pi(\mathrm{C} 9-\mathrm{C} 10)$ & $\pi^{*}(\mathrm{C} 10-\mathrm{C} 11)$ & 1.28 & 1.20 & 0.050 \\
\hline & $\pi(\mathrm{C} 10-\mathrm{C} 11)$ & $\pi^{*}(\mathrm{C} 1-\mathrm{C} 12)$ & 2.94 & 1.18 & 0.074 \\
\hline & $\pi(\mathrm{C} 11-\mathrm{C} 12)$ & $\pi^{*}(\mathrm{C} 9-\mathrm{C} 10)$ & 2.71 & 1.21 & 0.072 \\
\hline & $\sigma(\mathrm{C} 11-\mathrm{C} 12)$ & $\sigma^{*}(\mathrm{C} 1-\mathrm{C} 2)$ & 12.97 & 0.28 & 0.079 \\
\hline & & $\sigma^{*}(\mathrm{C} 4-\mathrm{C} 5)$ & 7.93 & 0.29 & 0.062 \\
\hline
\end{tabular}

\subsection{NLO analysis}

Non-linear optical (NLO) property arises from the interaction of applied electromagnetic fields in various materials [29]. The NLO response materials are providing the key functions for frequency shifting, optical switching, optical modulation, and optical logic for the developing technologies in areas such as communication, signal processing and optical interconnections [30]. The electric dipole moment $\mu$ and first order hyperpolarizability $\beta$ values of the molecular systems were based on the finite field approach. The energy of a system is a function of the applied electric field. The first order hyperpolarizability is a third rank tensor which can be described by a $3 \times 3 \times 3$ matrix. The 27 components of the 3D matrix can be reduced - because of the Kleinman symmetry [31] — to 10 components: $\beta_{x x x}, \beta_{x x y}, \beta_{x y y}, \beta_{y y y}, \beta_{x x z}, \beta_{x y z}, \beta_{y y z}, \beta_{x z z}, \beta_{y z z}$ and $\beta_{z z z}$.

The total static dipole moment given by $\mu$ and the mean first order hyperpolarizability $\beta$ are defined using the $x, y$ and $z$ components accordingly:

$$
\begin{aligned}
& \mu=\sqrt{\left.\mu_{x}^{2}+\mu_{y}^{2}+\mu_{z}^{2}\right)}, \\
& \beta=\sqrt{\left.\beta_{x}^{2}+\beta_{y}^{2}+\beta_{z}^{2}\right)},
\end{aligned}
$$

where

$$
\begin{aligned}
& \beta_{x}=\left(\beta_{x x x}+\beta_{x y y}+\beta_{x z z}\right), \\
& \beta_{y}=\left(\beta_{y y y}+\beta_{y z z}+\beta_{y x x}\right), \\
& \beta_{z}=\left(\beta_{z z z}+\beta_{z x x}+\beta_{z y y}\right) .
\end{aligned}
$$

Thus,

$$
\begin{aligned}
\beta & =\left[\left(\beta_{x x x}+\beta_{x y y}+\beta_{x z z}\right)^{2}\right. \\
& +\left(\beta_{y y y}+\beta_{y z z}+\beta_{y x x}\right)^{2} \\
& \left.+\left(\beta_{z z z}+\beta_{z x x}+\beta_{z y y}\right)^{2}\right]^{1 / 2}
\end{aligned}
$$

In the present investigation, the calculated dipole moment and first order hyperpolarizability are shown in Table III. The first order hyperpolarizability conversion factor is: $8.6393 \times 10^{-33}$. The higher dipole moment suggests the system is highly reactive. The dipole moment of S-Indacene is very low; it is due to the symmetric nature of the molecular system. After the adsorption on Ag atoms, results show an increase in a dipole moment and first order hyperpolarizability indicating a strongest adsorption interaction between S-Indacene and silver. The values are relatively much higher than those of urea (calculated first order hyperpolarizability value of urea with LANL2DZ basis set is $0.4135 \times 10^{-31}$ ), suggesting Ag-S-Indacene as a potential candidate for NLO devices. As compared with the $\beta$ value of the tetracene- $\mathrm{Ag}_{3}$ cluster, S-Indacene is found to be an effective NLO material [24].

\subsection{UV-Vis studies}

The absorption spectra of S-Indacene and Ag-S-Indacene simulated in a gas phase using TDDFT/B3PW91/LANL2DZ are shown in Fig. 7. UV-Vis spectroscopy is an important characterization of metal nanoparticles. The metal synthesized silver, gold nanoparticles interact 
TABLE III

The calculated dipole moment, polarizability and hyperpolarizability values of the S-Indacene and Ag-S-Indacene systems using B3PW91/LANL2DZ level of theory.

\begin{tabular}{c|c|c}
\hline \hline $\begin{array}{c}\text { NLO } \\
\text { Parameters }\end{array}$ & S-Indacene & Ag-S-Indacene \\
\hline$\mu_{x}$ & 0.0000 & 6.3170 \\
$\mu_{y}$ & 0.0000 & -1.1529 \\
$\mu_{z}$ & 0.0000 & 0.6436 \\
$\mu_{\text {tot }}$ & 0.0009 & 6.4536 \\
$\beta_{x x x}$ & 0.0000 & 439.9385 \\
$\beta_{x x y}$ & 0.0000 & -42.9623 \\
$\beta_{x y y}$ & 0.0000 & 129.1109 \\
$\beta_{y y y}$ & 0.0000 & -43.3237 \\
$\beta_{x x z}$ & -0.0169 & 34.6653 \\
$\beta_{x y z}$ & -0.0057 & -11.3657 \\
$\beta_{y y z}$ & -0.0025 & 0.7176 \\
$\beta_{x z z}$ & 0.0000 & 112.6776 \\
$\beta_{y z z}$ & 0.0000 & -7.4160 \\
$\beta_{z z z}$ & -0.0008 & 15.4209 \\
$\beta_{\text {tot }}$ & $1.7451 \times 10^{-34}$ & $5.9612 \times 10^{-30}$
\end{tabular}

strongly with light resulting in specific wavelength [32]. The absorption peak of S-Indacene appears around $474 \mathrm{~nm}$. After adsorption, the absorption peak of Ag-S-Indacene system was obtained as red-shifted at $714 \mathrm{~nm}$. The appearance of the red shift in the spectrum of the Ag-S-Indacene system evidences a charge transfer between the S-Indacene and $\mathrm{Ag}_{3}$ metal cluster. The appearance of the red shift identifies the aggregation of AgNPs confirming the process of adsorption of S-Indacene on AgNPs [5]. In addition to this, it also reflects $\pi \rightarrow \pi^{*}$ electronic transition in the visible region. This $\pi \rightarrow \pi^{*}$ electronic transition is responsible for the potential OLED application.

3.6. Vibrational analysis of S-Indacene and Ag-S-Indacene

In order to investigate the interaction between S-Indacene and Ag, DFT simulated Raman spectra of the molecular systems are shown in Fig. 8. The calculated vibrational wavenumbers with their corresponding assignments are listed in Table IV. Variations that occur in the vibrational modes and Raman intensities due to the surface adsorption of S-Indacene and Ag-S-Indacene cause the symmetry breaking. This, in turn, results in the excitation of additional vibrational modes. The peaks at lower frequencies are around $300 \mathrm{~cm}^{-1}$ for S-Indacene while for Ag-S-Indacene they change from $93 \mathrm{~cm}^{-1}$ to $400 \mathrm{~cm}^{-1}$, corresponding to metalmolecule stretching. The tilted orientation behaviour of the molecules on the metal surfaces can be determined on the basis of surface-enhanced Raman spectroscopy (SERS) selection rule [33, 34].
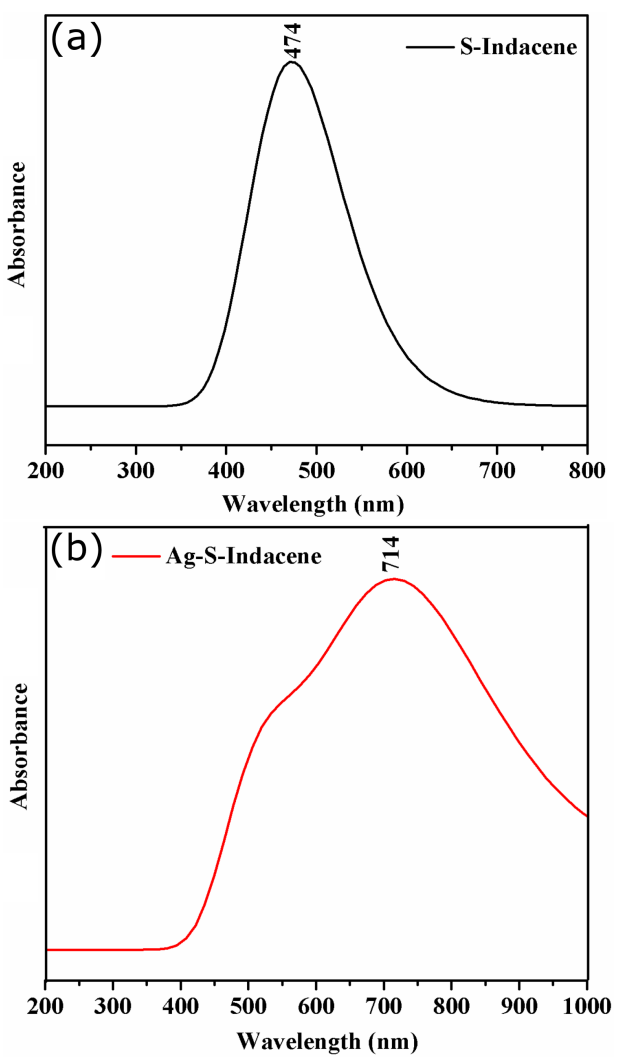

Fig. 7. UV-Vis absorption spectra of (a) S-Indacene and (b) Ag-S-Indacene systems.
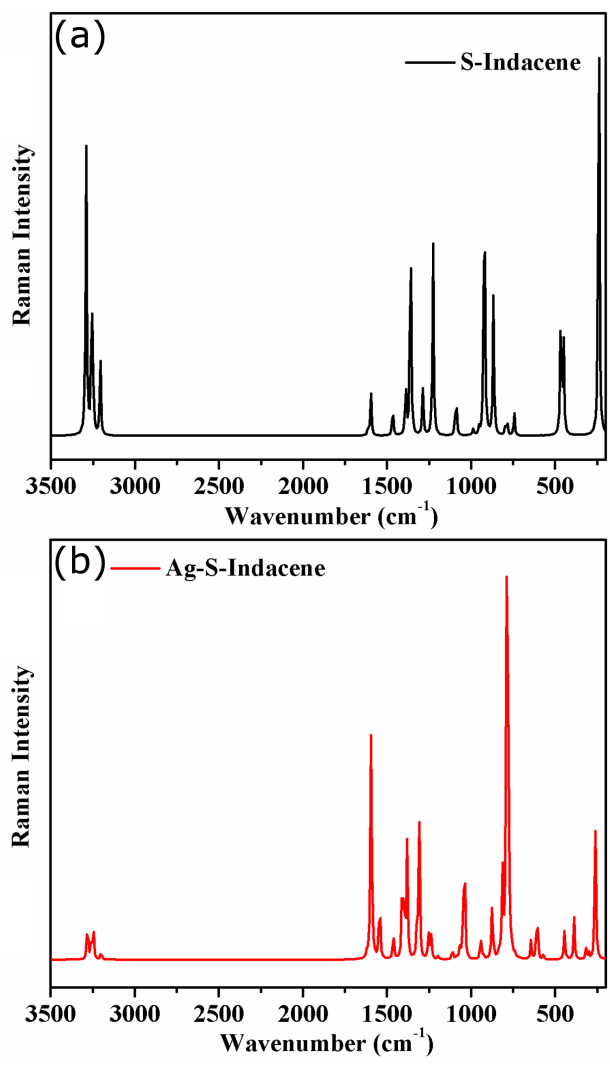

Fig. 8. The simulated (a) nRs spectrum of S-Indacene, and (b) SERS spectrum of Ag-S-Indacene system. 
TABLE IV

The calculated vibrational frequencies and their assignments of S-Indacene and Ag-S-Indacene systems, where $\nu$ means stretching, $\zeta$ is deformation, $\beta$ is in-plane deformation and $\gamma$ means out-of-plane deformation.

\begin{tabular}{|c|c|c|}
\hline \multicolumn{2}{|c|}{ Theoretical frequencies $\left[\mathrm{cm}^{-1}\right]$} & \multirow{2}{*}{ Assignments } \\
\hline S-Indacene & Ag-S-Indacene & \\
\hline- & 93 & $\nu \mathrm{I}-\mathrm{Ag}$ \\
\hline- & 108 & $\nu \mathrm{I}-\mathrm{Ag}$ \\
\hline- & 235 & $\nu \mathrm{I}-\mathrm{Ag}$ \\
\hline 321 & 365 & $\nu \mathrm{I}-\mathrm{Ag}$ \\
\hline- & 385 & $\nu \mathrm{I}-\mathrm{Ag}$ \\
\hline 508 & 400 & $\nu \mathrm{I}-\mathrm{Ag}$ \\
\hline- & 427 & $\zeta \mathrm{C}-\mathrm{C}-\mathrm{C}, \zeta \mathrm{C}-\mathrm{C}$ \\
\hline- & 526 & $\zeta \mathrm{C}-\mathrm{C}-\mathrm{C}, \zeta \mathrm{C}-\mathrm{C}$ \\
\hline- & 537 & $\zeta \mathrm{C}-\mathrm{C}-\mathrm{C}, \zeta \mathrm{C}-\mathrm{C}$ \\
\hline 637 & 650 & $\zeta \mathrm{C}-\mathrm{C}-\mathrm{C}, \zeta \mathrm{C}-\mathrm{C}$ \\
\hline 759 & 769 & $\zeta \mathrm{C}-\mathrm{C}-\mathrm{C}, \zeta \mathrm{C}-\mathrm{C}$ \\
\hline 840 & 820 & $\zeta \mathrm{C}-\mathrm{C}-\mathrm{C}, \zeta \mathrm{C}-\mathrm{C}$ \\
\hline- & 882 & $\zeta \mathrm{C}-\mathrm{C}-\mathrm{C}, \zeta \mathrm{C}-\mathrm{C}$ \\
\hline 985 & 942 & $\zeta \mathrm{C}-\mathrm{C}-\mathrm{C}, \zeta \mathrm{C}-\mathrm{C}$ \\
\hline 1001 & 961 & $\gamma \mathrm{C}-\mathrm{H}, \gamma \mathrm{C}-\mathrm{C}-\mathrm{H}$ \\
\hline 1012 & 1008 & $\gamma \mathrm{C}-\mathrm{H}, \gamma \mathrm{C}-\mathrm{C}-\mathrm{H}$ \\
\hline 1032 & 1018 & $\beta \mathrm{C}-\mathrm{H}, \beta \mathrm{C}-\mathrm{C}-\mathrm{H}$ \\
\hline 1056 & 1038 & $\beta \mathrm{C}-\mathrm{H}, \beta \mathrm{C}-\mathrm{C}-\mathrm{H}$ \\
\hline- & 1046 & $\beta \mathrm{C}-\mathrm{H}, \beta \mathrm{C}-\mathrm{C}-\mathrm{H}$ \\
\hline- & 1120 & $\beta \mathrm{C}-\mathrm{H}, \beta \mathrm{C}-\mathrm{C}-\mathrm{H}$ \\
\hline 1283 & 1252 & $\beta \mathrm{C}-\mathrm{H}, \beta \mathrm{C}-\mathrm{C}-\mathrm{H}$ \\
\hline- & 1286 & $\beta \mathrm{C}-\mathrm{H}, \beta \mathrm{C}-\mathrm{C}-\mathrm{H}$ \\
\hline 1420 & 1328 & $\beta \mathrm{C}-\mathrm{H}, \beta \mathrm{C}-\mathrm{C}-\mathrm{H}$ \\
\hline- & 1470 & $\nu \mathrm{C}=\mathrm{C}$ \\
\hline 1574 & 1596 & $\nu \mathrm{C}=\mathrm{C}$ \\
\hline 1629 & 1608 & $\nu \mathrm{C}=\mathrm{C}$ \\
\hline- & 1680 & $\nu \mathrm{C}=\mathrm{C}$ \\
\hline 3210 & - & $\nu \mathrm{C}-\mathrm{H}, \nu \mathrm{H}-\mathrm{C}-\mathrm{H}$ \\
\hline 3320 & - & $\nu \mathrm{C}-\mathrm{H}, \nu \mathrm{H}-\mathrm{C}-\mathrm{H}$ \\
\hline
\end{tabular}

Peaks identified at 427, 537, 769, 820 and 942 correspond to $\mathrm{C}-\mathrm{C}-\mathrm{C}$ stretching and bending vibrational modes suggesting that upon adsorption vibrations break the symmetry in the molecular systems resulting in a perpendicular orientation of S-Indacene on Ag [17-34]. Further, a significant enhancement upon the adsorption (Ag-S-Indacene: $1608,1650 \mathrm{~cm}^{-1}$ ) corresponds to $\mathrm{C}=\mathrm{C}$ stretching vibrations. It is identified that the corresponding enhanced peak in the Ag-S-Indacene spectra of the molecule is chemisorbed by the high affinity AgNP surface. The corresponding ring stretching modes observed at $1120,1252,1286,1328 \mathrm{~cm}^{-1}$ are red shifted. Thus, it is documented that red-shift denotes that S-Indacene is chemisorbed on the high affinity AgNP surface with a perpendicular orientation via $\sigma$ system of S-Indacene ring [35]. The vibrational ring stretching mode validates red shifting, suggesting a very strong interaction between the ring of organic moiety and silver surface. It is strongly confirmed from the finding that a charge transfer can also occur between molecule and metal surface with tilted orientation. Apart from this, the presence of a larger magnitude of red shifted $\mathrm{C}-\mathrm{H}, \mathrm{C}-\mathrm{C}-\mathrm{H}$ with in plane and out-of-plane vibrational modes results in breaking of the symmetry of the cyclopentane ring near the silver cluster accounting for the process of adsorption. This indicates that S-Indacene is attracted to the surface of silver through the cyclopentane ring. It is well correlated through molecular orbitals (MOs), molecular electrostatic potential (MEP) plot, natural bond orbitals (NBO) and natural population (NPA) analysis.

3.7. Fukui function analysis and local reactivity descriptors

for S-Indacene before and after adsorption

The Fukui analysis effectively identifies changes in electron density of the molecular system at a specific position, i.e., when donating or accepting electrons [36]. Among all atoms in the system, the atom with the highest value of Fukui function is considered to be the most reactive site [37]. As mentioned, the chemical reactivity of the molecular system is described by Fukui functions. They are defined as:

$$
f_{k}^{+}=q(N+1)-q(N)
$$

in the case of nucleophilic attack, as

$$
f_{k}^{-}=q(N)-q(N-1),
$$

in the case of electrophilic attack, while in the case of radial attack as

$$
f_{k}^{0}=\frac{1}{2}(q(N+1)-q(N-1)) .
$$

The higher Fukui function $f_{k}^{+}$describes the tendency of loss of an electron from an atom which corresponds to the favourable nucleophilic attack site. It also describes the tendency to accept an extra electron from an atom which corresponds to the favourable electrophilic attack site $f_{k}^{-}$and $f_{k}^{0}$ describes the favourable radical attack [36]. In the present work, Fukui calculations and the local reactivity descriptor were carried out at DFT/B3PW91/LANL2DZ level of theory. To predict the chemical reactivity of the molecular system for a particular site, we calculated the Fukui function $\left(f_{k}^{+}, f_{k}^{-}, f_{k}^{0}\right)$, based on the natural population analysis (NPA) with the charges $0,+1$ and -1 , namely neutral, cationic and anionic species. The values obtained for S-Indacene and Ag-S-Indacene were listed in Table $\mathrm{V}$ and Table VI, respectively. The highest $f_{k}^{+}$value of S-Indacene follows the order $\mathrm{C} 8>\mathrm{C} 10>\mathrm{C} 12=\mathrm{C} 2>\mathrm{C} 4>\mathrm{C} 6$ as 
TABLE V

The calculated Fukui function values of S-Indacene system.

\begin{tabular}{l|c|c|c}
\hline \hline \multirow{2}{*}{ Atom } & \multicolumn{3}{|c}{ S-Indacene } \\
\cline { 2 - 4 } & $f_{k}^{0}$ & $f_{k}^{+}$ & $f_{k}^{-}$ \\
\hline C1 & 0.0796 & 0.0320 & -0.1817 \\
C2 & 0.0892 & 0.1549 & 0.0215 \\
C3 & 0.0796 & 0.0150 & 0.1442 \\
C4 & 0.0351 & 0.0834 & -0.0132 \\
C5 & 0.0520 & 0.0000 & 0.1000 \\
C6 & 0.0351 & 0.0761 & -0.0058 \\
C7 & 0.0796 & 0.0320 & 0.1271 \\
C8 & 0.0882 & 0.1549 & 0.0215 \\
C9 & 0.0796 & 0.0150 & 0.1442 \\
C10 & 0.0351 & 0.0834 & -0.0132 \\
C11 & 0.0521 & 0.0039 & 0.1000 \\
C12 & 0.0351 & 0.0761 & -0.0058 \\
H13 & 0.0327 & 0.0346 & 0.0309 \\
H14 & 0.0318 & 0.0304 & 0.0331 \\
H15 & 0.0327 & 0.0351 & 0.0303 \\
H16 & 0.0328 & 0.0341 & 0.0315 \\
H17 & 0.0327 & 0.0346 & 0.0309 \\
H18 & 0.0320 & 0.0304 & 0.0336 \\
H19 & 0.0303 & 0.0351 & 0.0303 \\
H20 & 0.0315 & 0.0341 & 0.0315 \\
& & &
\end{tabular}

compared to the other carbon atoms C1, C3, C5, $\mathrm{C} 7, \mathrm{C} 9, \mathrm{C} 11$. This result indicates that the carbon atoms $(\mathrm{C} 8, \mathrm{C} 10 \ldots \mathrm{C} 12)$ near to the Ag cluster contribute more to be the most reactive sites for nucleophilic attack than the hydrogen atoms. The highest $f_{k}^{-}$value indicates that the carbon atoms C3, $\mathrm{C} 7$ contribute much to be the most reactive sites for electrophilic attack. In the case of Ag-S-Indacene, the highest $f_{k}^{-}$value of $\mathrm{Ag}$ atoms varies significantly, clearly explaining the reliable charge transfer from molecule to metal.

The electronic chemical potential $\mu$, the absolute hardness $\eta$ and the global electrophilicity index $\omega$ are defined as:

$$
\begin{aligned}
\mu & =\frac{1}{2}(I+A) \\
\eta & =\frac{1}{2}(I-A) \\
\omega & =\frac{\mu^{2}}{2 \eta},
\end{aligned}
$$

where $I$ is the vertical ionization potential and $A$ is the vertical electron affinity. The ionization potential is calculated as $I=E(N-1)-E(N)$. The electron affinity is calculated as $A=E(N+1)-E(N)$, where $E(N)$ and $E(N+1)$ are the total ground state energies in the neutral $\mathrm{N}$ and singly charged $(N+1)$ configurations. The theoretically calculated
TABLE VI

\begin{tabular}{|c|c|c|c|}
\hline \multirow{2}{*}{ Atom } & \multicolumn{3}{|c|}{ Ag-S-Indacene } \\
\hline & $f_{k}^{0}$ & $f_{k}^{+}$ & $f_{k}^{-}$ \\
\hline $\mathrm{C} 1$ & 0.1012 & 0.1015 & 0.1009 \\
\hline $\mathrm{C} 2$ & 0.0094 & 0.0164 & 0.0024 \\
\hline $\mathrm{C} 3$ & 0.0994 & 0.1013 & 0.0975 \\
\hline $\mathrm{C} 4$ & 0.0093 & -0.0195 & 0.0008 \\
\hline C5 & 0.0595 & 0.0962 & 0.0229 \\
\hline C6 & -0.0731 & -0.0214 & 0.0068 \\
\hline $\mathrm{C} 7$ & 0.0924 & 0.1875 & -0.0027 \\
\hline $\mathrm{C} 8$ & -0.0255 & -0.0639 & 0.0128 \\
\hline C9 & 0.0347 & 0.0732 & -0.0037 \\
\hline $\mathrm{C} 10$ & -0.0048 & -0.0152 & 0.0054 \\
\hline $\mathrm{C} 11$ & 0.0572 & 0.0906 & 0.0239 \\
\hline $\mathrm{C} 12$ & -0.0110 & -0.0170 & -0.0507 \\
\hline $\mathrm{H} 13$ & 0.0203 & 0.0252 & 0.0154 \\
\hline H14 & 0.0236 & 0.0262 & 0.0181 \\
\hline H15 & 0.0202 & 0.0254 & 0.0150 \\
\hline H16 & 0.0176 & 0.0222 & 0.0129 \\
\hline $\mathrm{H} 17$ & 0.0068 & 0.0027 & 0.0106 \\
\hline H18 & 0.0159 & 0.0214 & 0.0104 \\
\hline H19 & 0.0145 & 0.0179 & 0.0110 \\
\hline $\mathrm{H} 20$ & 0.0182 & 0.2210 & 0.0144 \\
\hline Ag21 & 0.2337 & 0.1470 & 0.3203 \\
\hline $\operatorname{Ag} 22$ & 0.1875 & 0.1104 & 0.2646 \\
\hline Ag23 & 0.0454 & 0.0490 & 0.4170 \\
\hline
\end{tabular}

The calculated Fukui function values of Ag-S-Indacene system.

TABLE VII

Local reactivity descriptors of S-Indacene and Ag-S-Indacene systems.

\begin{tabular}{c|c|c}
\hline \hline $\begin{array}{c}\text { Molecular } \\
\text { properties }\end{array}$ & S-Indacene $[\mathrm{eV}]$ & Ag-S-Indacene $[\mathrm{eV}]$ \\
\hline$I$ & 7.20 & 5.53 \\
$A$ & -1.70 & -1.58 \\
$\mu$ & -2.74 & -1.97 \\
$\eta$ & -4.45 & -3.56 \\
$\omega$ & -0.84 & -0.54
\end{tabular}

chemical potential, electron affinity and electrophilicity index of S-Indacene and Ag-S-Indacene systems was listed in Table VII. The global reactivity descriptor clearly describes the inter- and intramolecular reactivity of the molecular system as the product of the Fukui function and global electrophilicity index. When two molecular systems interact with each other, the ability of a molecular system to accept electrons refers to higher electrophilic index and the ability of a molecular 
system to donate electrons refers to higher nucleophilic index [37]. From the theoretical results, predicted in Table VII, it is identified that the chemical potential of S-Indacene is greater after the adsorption and there is a reduction in the global hardness and electrophilic index $(\omega)$. This indicates S-Indacene is prone to be more nucleophilic, resulting in the flow of electrons from S-Indacene to the silver cluster associated with a strong surface interaction between molecule and metal.

\section{Conclusion}

Spectroscopic and charge transfer analysis of S-Indacene on silver is studied using DFT approach. The smallest $\lambda_{e}$ confirms the application of the molecule as ETL in OLED devices. The interfacial charge transfer has been identified from the shifting of frontier molecular orbitals. It is further confirmed by MEP plot, NBO, NPA and Fukui calculations. The adsorption is further validated by UV-Vis and Raman shifts. Investigation of the vibrational analysis confirms the adsorption and occurrence of a charge transfer under tilted orientation of the molecule on the silver surface. The metal-molecule adsorption interaction is between $\sigma$ of S-Indacene and valence orbitals of silver. The distortion in the cyclopentane ring near to the silver cluster is identified after the adsorption along with deviations in the planar cyclic conjugated symmetry of S-Indacene. The reduction in band gap and enhancement in first order hyperpolarizability reveals Ag-S-Indacene can be used in the design of electro-active organic semiconducting devices and novel NLO materials.

\section{References}

[1] D. Sajan, K. Chaitanya, K. Safakath, R. Philip, T.Suthan, N.P. Rajeshd, Spectrochim. Acta A 106, 253 (2013).

[2] M. Nendel, B. Goldfuss, K.N. Houk, K. Hafner, J. Mol. Struct. (Theochem) 461, 23 (1999).

[3] K.L. Kelly, E. Coronado, L.L. Zhao, G.C. Schatz, J. Phys. Chem. B 107, 668 (2003).

[4] V.E. Matulis, A.S. Mazheika, O.A. Ivashkevich, J. Mol. Struct. (Theochem) 850, 61 (2008).

[5] T.N. Rekha, M. Umadevi, B.J.M. Rajkumar, Mol. Phys. 113, 3673 (2015).

[6] U.R. Felscia, B.J.M. Rajkumar, M.B. Mary, Synth. Met. 246, 31 (2018).

[7] K. Hafner, K.H. Hafner, C. Konig, M. Kreuder, G. Ploss, G. Schulz, E. Sturm, K.H. Vöpel, Angew. Chem. Int. Ed. 2, 123 (1963).
[8] X. Zarate, E. Schott, E. Bunel, J.M. Manquez, I. Chavez, J. Chem. 2017, 9101720 (2017).

[9] J.-P. Jalkanen, F. Zerbetto, J. Phys. Chem. B 110, 5595 (2006).

[10] T. Woldu, B. Raneesh, P. Sreekanth, M.V.R. Reddy, R. Philip N. Kalarikkalce, J. Phys. Chem. Lett. 625, 58 (2015).

[11] A. Shimizu, Y. Tobe, Angew. Chem. Int. Ed. 50, 6906 (2011).

[12] Xueliang Shi, P. Mayorga Burrezo, S. Lee et al. Chem. Sci. 5, 4490 (2014).

[13] N. Kalashnyk, K. Mouhat, J. Oh, J. Jung, Y. Xie, E. Salomon, T. Angot, F. Dumur, D. Gigmes S. Clair, Nat. Commun. 8, 14735 (2017).

[14] V. Butera, M.C. Toroker, Materials 10, 480 (2017).

[15] J. Paier, M. Marsman, G. Kresse, J. Chem. Phys. 127, 024103 (2007).

[16] M.J. Huang, J.D. Watts, Phys. Chem. Chem. Phys. 14, 6849 (2012).

[17] C. Gellini, G. Cardini, P.R. Salvi, G. Marconi, K. Hafner, J. Phys. Chem. 97, 1286 (1993).

[18] U. Reeta Felscia, B.J.M. Rajkumar, P. Sankar, R. Philip, M.B. Mary, Spectrochim. Acta A 184, 286 (2017).

[19] S. Premkumar, T.N. Rekha, R.M. Asath, T. Mathavan, A. Milton, F. Benial, Eur. J. Pharm. Sci. 82, 115 (2016).

[20] B. Babu, J. Chandrasekaran, B. Mohanbabu, Y. Matsushita, M. Saravanakumar, RSC Adv. 6, 110884 (2016).

[21] V. Narayan, H.N. Mishra, O. Prasad, L. Sinha, Comput. Theor. Chem. 973, 20 (2011).

[22] D. Tuncela, Nanoscale Adv. 1, 19 (2019).

[23] T.N. Rekha, M. Umadevi, B.J.M. Rajkumar, J. Mol. Struct. 1079, 155 (2015).

[24] T.N. Rekha, Beulah J.M. Rajkumar, Synth. Met. 215, 207 (2016).

[25] T. Karthick, V. Balachandran, S. Perumal, A. Nataraj, J. Mol. Stru. 1005, 202 (2011).

[26] M. Karabacak, M. Cinar, M. Kurt, A. Poiyamozhi, N. Sundaraganesan, Spectrochim. Acta A 117, 234 (2014).

[27] N.R. Sheela, S. Muthu, S. Sampathkrishnan, Spectrochim. Acta A 120, 237 (2014).

[28] L. Jun-na, C. Zhirang, Y. Shen-Fang, J. Zhejiag Univ. Sci. B 6, 584 (2005).

[29] T. Rajamani, S. Muthu, M. Karabacak, Spectrochim. Acta A 108, 186 (2013). 
[30] M. Khalid, R. Hussain, A. Hussain et al. Molecules 24, 2096 (2019).

[31] D.A. Kleinman, Phys. Rev. 126, 1977 (1962).

[32] K. Geetha, M. Umadevi, G.V. Sathe, R. Erenler, Spectrochim. Acta A 116, 236 (2013).

[33] E.C. Le Ru, S.A. Meyer, C. Artur, P.G. Etchegoin, J. Grand, P. Lang, F. Maurel, Chem. Commun. 47, 3903 (2011).
[34] U. Reeta Felscia, B.J.M. Rajkumar, Mater. Lett. 221, 318 (2018).

[35] R. Premkumar, S. Hussain, T. Mathavan, K. Anitha, A.M.F. Benial, J. Mol. Liq. 290, 111209 (2019).

[36] R.K. Roy, S. Krishnamurthi, P. Geerlings, S. Pal, J. Phys. Chem. 102, 3746 (1998).

[37] J. Padmanabhan, R. Parthasarathi, U. Sarkar, V. Subramanian, P.K. Chattaraj Chem. Phys. Lett. 383, 122 (2004). 